\title{
Circumferential vascular strain rate to estimate vascular load in aortic stenosis: a speckle tracking echocardiography study
}

\author{
Rogério Teixeira $\cdot$ Ricardo Monteiro $\cdot$ Rui Baptista $\cdot$ \\ António Barbosa • Luís Leite - Miguel Ribeiro • Rui Martins • \\ Nuno Cardim • Lino Gonçalves
}

Received: 12 August 2014/Accepted: 17 January 2015/Published online: 23 January 2015

(C) Springer Science+Business Media Dordrecht 2015

\begin{abstract}
Evaluation of vascular mechanics through twodimensional speckle-tracking (2D-ST) echocardiography is a feasible and accurate approach for assessing vascular stiffening. Degenerative aortic stenosis (AS) is currently considered a systemic vascular disease where rigidity of arterial walls increases. To assess the circumferential ascending aorta strain rate (CAASR) in thoracic aortas of patients with AS, applying 2D-ST technology. 45 patients with indexed aortic valve areas (iAVA) $\leq 0.85 \mathrm{~cm}^{2} / \mathrm{m}^{2}$ were studied. Global CAASR served to assess vascular deformation. Clinical, echocardiographic, and non-invasive hemodynamic data were collected. A follow up (955 days) was also performed. Average age of the cohort was 76. \pm 10.3 years, with gender balance. Mean iAVA was $0.43 \pm 0.15 \mathrm{~cm}^{2} / \mathrm{m}^{2}$. Waveforms adequate for determining CAASR were found in $246(91 \%)$ of the 270 aortic
\end{abstract}

Electronic supplementary material The online version of this article (doi:10.1007/s10554-015-0597-y) contains supplementary material, which is available to authorized users.

R. Teixeira $(\bowtie) \cdot$ R. Monteiro $\cdot$ M. Ribeiro

Departamento de Medicina, Serviço de Cardiologia, Hospital

Beatriz Ângelo, Avenida Carlos Teixeira - 3, 2674-514 Loures,

Portugal

e-mail: rogeriopteixeira@gmail.com

R. Teixeira $\cdot$ R. Baptista $\cdot$ L. Gonçalves

Faculdade de Medicina Universidade de Coimbra, Coimbra, Portugal

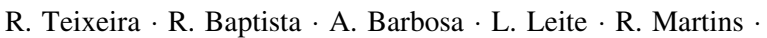
L. Gonçalves

Serviço de Cardiologia, Centro Hospitalar e Universitário de

Coimbra, Coimbra, Portugal

N. Cardim

Serviço de Cardiologia, Hospital da Luz, Lisbon, Portugal segments evaluated, for a mean global CAASR of $0.74 \pm 0.26 \mathrm{~s}^{-1}$. Both intra- and inter-observer variability of global CAASR were deemed appropriate. CAASR correlated significantly with age $(r=-0.49, p<0.01)$, the stiffness index $(r=-0.59, p<0.01)$, systemic arterial compliance and total vascular resistance. There was a significant positive correlation between CAASR, body surface area (BSA), iAVA, and a negative relationship with valvulo-arterial impedance and E/e' ratio $(r=-0.37$, $p=0.01)$. The stiffness index was $(\beta=-0.41, p<0.01)$ independently associated with CAASR, in a model adjusted for age, BSA, iAVA and E/e'. Patients with a baseline CAASR $\leq 0.66 \mathrm{~s}^{-1}$ had a worse long-term outcome (survival 52.4 vs. $83.3 \%, \log \operatorname{Rank} p=0.04$ ). CAASR is a promising echocardiographic tool for studying the vascular loading component of patients with AS.

Keywords 2D-ST echocardiography - Aortic stenosis . Vascular mechanics · Vascular stiffness · Prognosis

\section{Introduction}

Degenerative calcific aortic stenosis (AS) is currently viewed as a complex, multifaceted and systemic disease [1], displaying atherosclerotic-like and elastocalcinosislike vascular changes that increase arterial wall rigidity [2]. Thus AS is not limited to valvular disease [2]. Arterial compliance is also reduced, and left ventricular (LV) geometry and function are altered [3].

Although the vascular component of AS is utmost importance, there is currently no gold standard method for determining local arterial stiffness. Available non-invasive methods show considerable differences in validity and reproducibility $[4,5]$. Surrogates for arterial stiffness may 
be derived non-invasively from pulse transit time, arterial pressure waves, or relational changes in vessel diameter and distending pressure. The latter may be expressed as distensibility, compliance, elastic modulus, or stiffness index $\left(\beta_{1}\right)[6]$.

Two-dimensional speckle-tracking (2D-ST) echocardiography involves identification of specific acoustic markers (i.e., speckles) in grey-scale images, tracking them frameby-frame throughout the cardiac cycle. This enables angleindependent calculations of motion and deformation variables, such as velocity, displacement, strain $(\varepsilon)$, and strain rate (SR). A number of speckle-tracking algorithms have been developed, albeit aimed primarily at cardiac applications [7, 8]. Since 2008, 2D-ST studies have proved successful in assessing local vascular wall properties of proximal elastic arteries [9-11]. Apart from circumferential vascular $\varepsilon$, the rate of deformation, named the circumferential SR is other published index of vascular stiffening and aging [9].

The current study was designed to: (1) assess circumferential ascending aorta strain rate (CAASR) using 2DST echocardiography in patients with moderate to severe degenerative AS; (2) to identify predictors of CAASR; (3) to analyze the association of CAASR with LV afterload variables; (4) finally to study the CAASR prognostic significance.

\section{Methodology}

A total of 53 consecutive patients referred for echocardiography in a single laboratory were enrolled for a 2-month study, between January and February 2012. Each patient had a calculated aortic valve area $\leq 0.85 \mathrm{~cm}^{2} / \mathrm{m}^{2}$. Eight patients were eliminated due to poor-quality images. The final cohort consisted of 45 patients with moderate to severe AS, as previously detailed [12].

Informed consent was obtained from all participants. The local ethics committee approved this protocol.

Clinical data, systemic arterial hemodynamics and follow-up

Data recorded for each enrollee at admission included age, weight, height, and medical conditions (diabetes, hypertension, and congestive heart failure). The body surface area (BSA) was estimated according to the formula by DuBois and DuBois [13].

Systemic arterial pressure was measured using an arm cuff sphygmomanometer (right brachial artery) simultaneously with Doppler measurement of left ventricular outflow tract (LVOT) stroke volume. Indexed systemic arterial compliance
(SAC) was calculated as follows: $\mathrm{SAC}=\mathrm{SVI} / \mathrm{PP}$, where SVI is stroke volume index and PP is brachial pulse pressure. A low state of compliance was defined as $\mathrm{SAC} \leq 0.6 \mathrm{ml} / \mathrm{mmHg}$ / $\mathrm{m}^{2}$ [2]. Total vascular resistance (TVR) was estimated as follows: TVR $=80 \times \mathrm{MAP} / \mathrm{CO}$, where MAP is mean arterial pressure (i.e., diastolic pressure plus one-third brachial pulse pressure) and CO is cardiac output [14]. Elevated TVR was defined as SVR $>2,000$ dynes $/ \mathrm{sec} / \mathrm{cm}^{-5}$ [2].

In November 2014, a clinical follow-up was performed by LL, who was blinded to the standard and advanced echocardiographic data. The following outcomes were analysed: all cause mortality; cardiovascular mortality; aortic valve replacement (AVR); and heart failure hospitalization due to AS. We also assessed a combined endpoint of mortality + AVR + heart failure hospitalization.

\section{Echocardiography}

A Vivid 7 (GE Healthcare ${ }^{\circledR}$, Horton, Norway) cardiovascular ultrasound device was used, with a $1.7 / 3.4 \mathrm{MHz}$ tissue harmonic transducer. Complete echocardiographic studies called for standard views and techniques stipulated by established guidelines [15]. In addition, short-axis views of ascending aorta, past sinotubular junction (usually 2-3 cm above aortic valve), were obtained at a high frame rate (mean value, $71.1 \pm 5.3 / \mathrm{s}$ ). For this purpose, machine settings were manually adjusted to optimize $2 \mathrm{D}$ aortic wall tracings and 2D-ST gray-scale definition. All images were acquired at end-expiratory apnea. Loops of three cardiac cycles were stored digitally and analyzed offline via custom software (EchoPAC 9.0, GE Healthcare ${ }^{\circledR}$, Horton, Norway).

\section{Left ventricular assessment}

Linear measurements of interventricular septum and posterior LV wall thickness and internal LV dimensions were acquired through a 2D long-axis parasternal window, in accord with accepted guidelines [16]. LV mass was calculated using a corrected formula of the American Society of Echocardiography and indexed for BSA [16].

Left ventricular end-systolic and end-diastolic volumes and LV ejection fraction (LVEF) were assessed using the modified Simpson's rule (method of disks) [16]. LV cardiac index was calculated as the product of heart rate and indexed stroke volume for BSA. Stroke volume was obtained by LV outflow Doppler method as the product of LVOT area and LVOT time-velocity integral [17]. E/e' ratio ( $\mathrm{e}$ ' being an average of septal and lateral walls in tissue Doppler imaging) was used to estimate LV filling pressures [18]. 
Global LV afterload, elastic properties of aorta, and severity of aortic valvular stenosis

Valvuloarterial impedance $\left(Z_{\mathrm{VA}}\right)$, as a measure of global LV afterload, was calculated as follows: $\mathrm{Z}_{\mathrm{VA}}=\mathrm{SAP}+\mathrm{MG} /$ $\mathrm{SVI}$, where SAP is systolic arterial pressure and MG is mean transvalvular pressure gradient [2].

Aortic distensibility (D) and stiffness index $\left(\beta_{1}\right)$ were calculated as follows: $\mathrm{D}=2\left(\mathrm{~A}_{\mathrm{s}}-\mathrm{A}_{\mathrm{d}}\right) /\left[\mathrm{A}_{\mathrm{d}}\left(\mathrm{P}_{\mathrm{s}}-\mathrm{P}_{\mathrm{d}}\right)\right]$ in $\mathrm{cm}^{2}$ dyne ${ }^{-1} 10^{-6}$ and $\beta_{1}=\ln \left(P_{s} / P_{d}\right) /\left(A_{s}-A_{d}\right) / A_{d}$ [19], where $P_{s}$ and $P_{d}$ are systolic and diastolic arterial pressures, and $A_{s}$ and $\mathrm{A}_{\mathrm{d}}$ are $\mathrm{M}$-mode guided systolic and diastolic ascending aortic diameters, 2-3 cm above aortic valve. $A_{d}$ was obtained as $R$ wave peaked in simultaneously recorded electrocardiogram, and $\mathrm{A}_{\mathrm{s}}$ was measured at maximal anterior aortic wall motion.

Classic Doppler echocardiographic indices of AS severity were assessed as well, including transvalvular (peak and mean) pressure gradients (by modified Bernoulli equation), indexed aortic valve area (iAVA) by continuity equation, and dimensionless velocity index (as ratio of LVOT time-velocity integral to aortic jet time-velocity integral). Energy loss index (ELI) was determined as follows: (AVA $\times \mathrm{AA} / \mathrm{AA}-\mathrm{AVA}) / \mathrm{BSA}$, where $\mathrm{AA}$ is aortic cross-sectional area at level of sinotubular junction [20].

\section{Two-dimensional speckle-tracking strain echocardiography}

As in a prior publication of ours [12], calculations of regional and global thoracic ascending aortic mechanics relied on 2D-ST technology. With a line manually drawn along the inner aspect of aortic wall in short axis, additional lines were automatically generated (via 2D-ST) at the outer aspect of vessel wall. Considering the relative thinness of vascular walls (compared with cardiac walls), region of interest width was reduced to the minimal value allowable by software, as previously suggested [21]. The initial systolic frame generally served as the frame of interest, to include maximal aortic wall expansion and recoil. As in other instances $[10,22]$, aortic wall was divided into six equidistant regions, all similar in size. In each region, numeric expressions of each 2D-ST variable represented mean values calculated from all points in arterial segments. These were color-coded and shown as a function of time throughout the cardiac cycle. Quantitative curves, depicting all regions, were possible for each $2 \mathrm{D}-\mathrm{ST}$ variable. The tracking process and conversion to Lagrangian strains were performed offline, using dedicated software. CAASR curves generated here were aligned with those generated elsewhere [6, 9] and included a positive early systolic peak. Global CAASR was then calculated as the mean of peak values for the six segments (Fig. 1).
For the follow-up analysis we used data from our prior publication [12], regarding the global circumferential ascending aortic strain (CAAS).

We have also analyzed the LV global longitudinal $\varepsilon$ with the 2D-ST. We calculated a mean value of 18 myocardial segments, 6 from each of the three standard apical views as previously reported [23].

\section{Statistical analysis}

The Kolmogorov-Smirnov test was used to confirm normal distribution of all continuous variables, expressed as mean and standard deviation. Student's $t$ test or Anova were applied for group comparisons. Individual variables were checked for homogeneity of variance via Levene's test. Categorical variables were reported as frequencies and percentages, and $\chi^{2}$ or Fisher exact tests were used when appropriate.

Based on stored images of 15 randomly selected patients, intra- and inter-observer reproducibility of CAASR values were assessed by Bland-Altman method [24] and intra-class correlation coefficient (ICC) [25].

Pearson's correlation was used to analyze the relationship between CAASR and an array of continuous variables. A linear regression analysis was performed thereafter to identify independent predictors of CAASR. We created three different models, one with clinical data, one with afterload data, and one with valvular plus LV data. A final multivariate model including clinical, afterload and LV data was subsequently elaborated. Variables identified as significant on the bivariate analysis $(p<0.05)$ and with clinical relevance, were included in the model.

A receiver-operating characteristic (ROC) curve analysis was used to compute the discriminatory power of CAASR to predict survival. The cumulative survival curves were constructed using the Kaplan-Meier method, and the groups were compared with the Log-Rank test.

A $p$ value $<0.05$ in two-tailed tests was considered statistically significant. All data calculations and analyses relied on SPSS ${ }^{\circledR} 15$, Medcalc ${ }^{\circledR}$ 12.1.4 and GraphPad Prism ${ }^{\circledR} 6.05$ statistical software packages.

\section{Results}

Average age of the 45 patients studied was $76.8 \pm 10.3$ years, with gender balance. Mean iAVA was $0.43 \pm 0.15 \mathrm{~cm}^{2} / \mathrm{m}^{2}$.

Waveforms adequate for measuring CAASR were present in $246(91 \%)$ of the 270 arterial segments evaluated. Mean global CAASR was $0.74 \pm 0.26 \mathrm{~s}^{-1}$ (Table 1).

Circumferential ascending aorta strain rate correlated significantly with age $(r=-0.49, p<0.01)$, BSA, and pulse pressure. It also showed significant associations with systemic arterial hemodynamic and aortic elastic variables 
Fig. 1 Global CAASR $\left(\mathrm{s}^{-1}\right)$ generated from short axis view of aorta, 2-3 cm above aortic valve. (a) Thoracic ascending aorta region of interest (short axis view). (b) Color M-mode of CAASR for all regions during cardiac cycle. (c) Color-coded curves of defined aortic segment (depicted in figure); global CAASR indicated by white dotted curve. Circumferential SR (first peak after ventricular systole) assumes early positive value due to vessel wall expansion

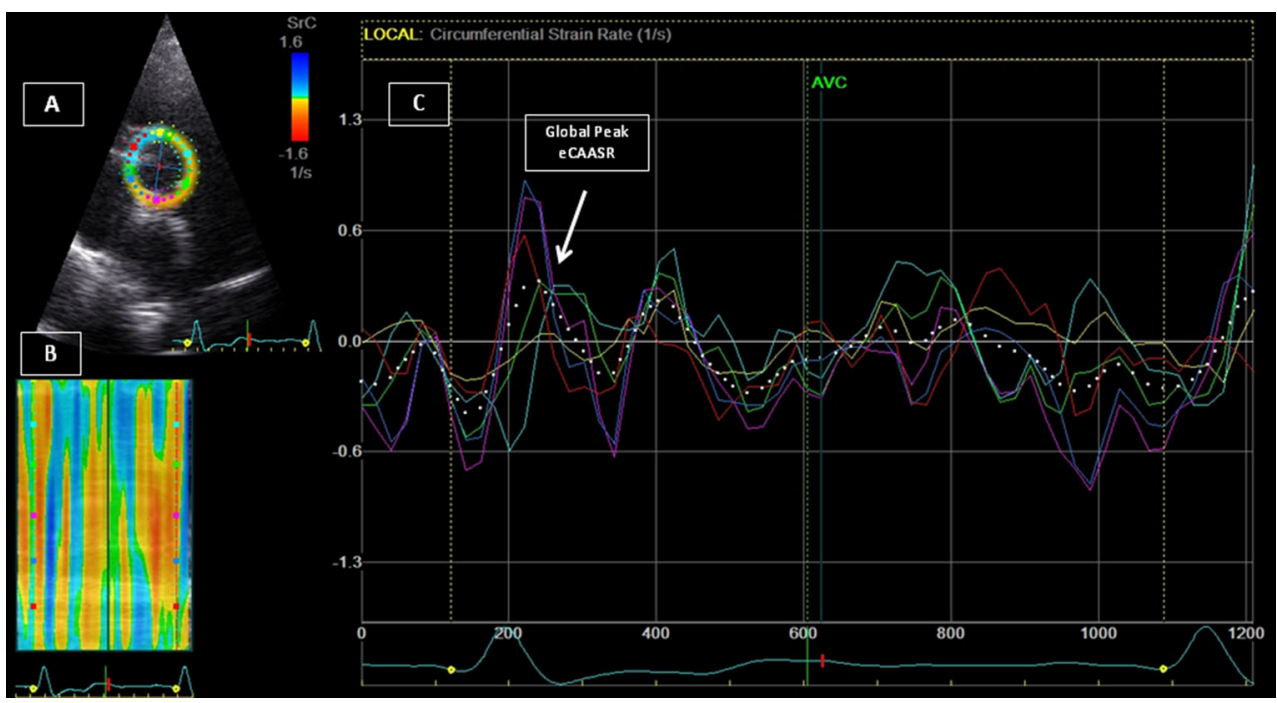

such as SAC $(r=0.54, p<0.01)$ (Fig. 2, Panel A), TVR $(r=-0.49, p<0.01)$, and $\beta_{1}(r=-0.59, p<0.01)$ (Fig. 2, Panel B).

There was a significant positive correlation between CAASR and iAVA $(r=0.44, p<0.01)$ and a negative correlation with $\mathrm{Z}_{\mathrm{VA}}(r=-0.59, p<0.01)$.

With respect to LV performance variables, global CAASR correlated significantly with SVI $(r=0.36, p=0.02)$, with LVEF, and with E/e' ratio ( $r=-0.37, p=0.01)$ (Table 2$)$.

We created three multivariate models to predict CAASR, based on clinical (Table 3), afterload (Table 4) and on valvular plus LV data (Table 5). We then constructed a new model that included the most relevant variables from each previous model. We demonstrated that the stiffness index was $(\beta=-0.41, p<0.01)$ independently associated with CAASR, when adjusted for age, BSA, iAVA and estimated LV filling pressures (Table 6). This model had the highest $R^{2}(0.57)$ of all.

Agreement and reproducibility

Intra-observer variability of CAASR was $0.01 \mathrm{~s}^{-1}(95 \%$ confidence interval [CI] 0.08-0.1 s ${ }^{-1}$ ) (Fig. 3, Panel A).

Table 1 Circumferential ascending aorta strain rate

\begin{tabular}{ll}
\hline Total population $(n=45)$ & \\
\hline Global CAASR $\left(\mathrm{s}^{-1}\right)$ & $0.74 \pm 0.26$ \\
Segment 1 CAASR $\left(\mathrm{s}^{-1}\right)$ & $0.57 \pm 0.39$ \\
Segment 2 CAASR $\left(\mathrm{s}^{-1}\right)$ & $0.74 \pm 0.32$ \\
Segment 3 CAASR $\left(\mathrm{s}^{-1}\right)$ & $0.83 \pm 0.39$ \\
Segment 4 CAASR $\left(\mathrm{s}^{-1}\right)$ & $0.83 \pm 0.39$ \\
Segment 5 CAASR $\left(\mathrm{s}^{-1}\right)$ & $0.78 \pm 0.43$ \\
Segment 6 CAASR $\left(\mathrm{s}^{-1}\right)$ & $0.68 \pm 0.36$
\end{tabular}

CAASR circumferential ascending aortic strain rate
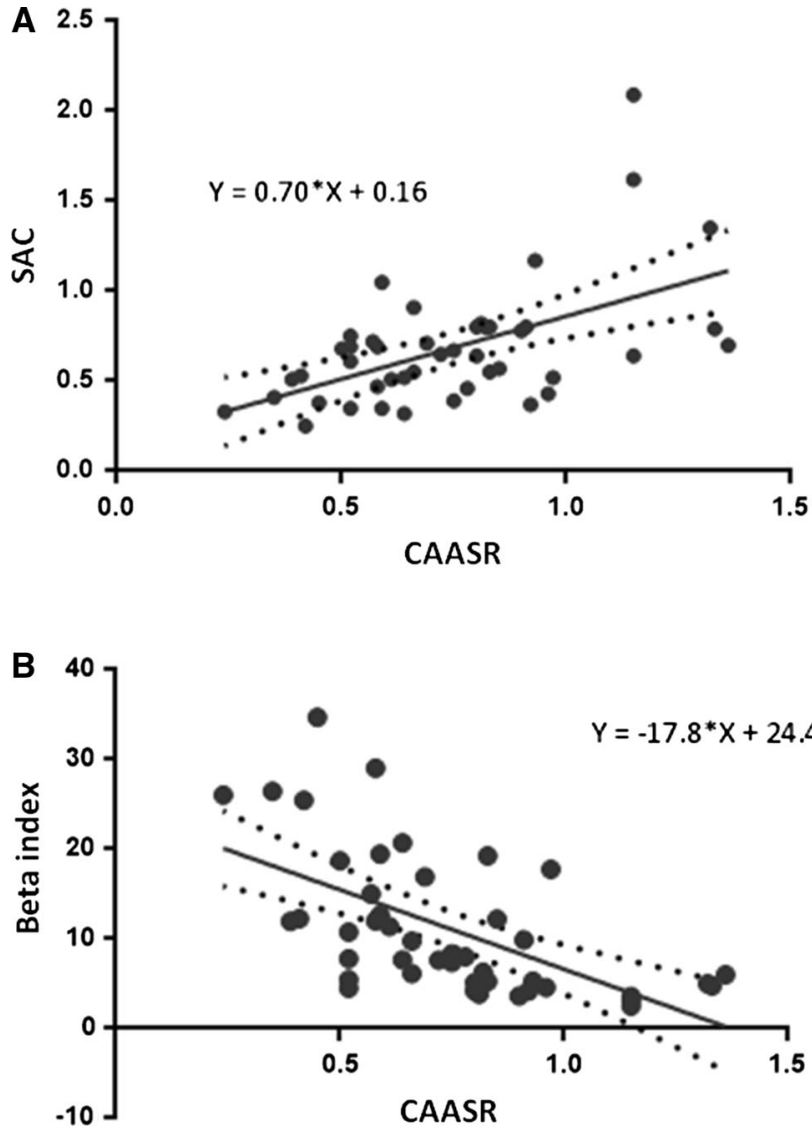

Fig. 2 a Correlation between global CAASR and SAC, b Correlation between global CAASR and $\beta_{1}$

The ICC of intra-observer CAASR variability was 0.97 (95\% CI 0.93-0.99).

Inter-observer variability of CAASR was $-0.02 \mathrm{~s}^{-1}$ (95\% CI 0.16-0.11 s ${ }^{-1}$ ) (Fig. 3, Panel B). The ICC of inter-observer CAASR variability was 0.97 (95\% CI 0.91-0.98). 
Table 2 Correlations of circumferential ascending aorta strain rate

\begin{tabular}{|c|c|c|}
\hline \multirow[t]{2}{*}{ Clinical variables } & \multicolumn{2}{|c|}{ CAASR } \\
\hline & $r$ & $p$ \\
\hline Age (years) & -0.49 & $<0.01$ \\
\hline Body surface area $\left(\mathrm{m}^{2}\right)$ & 0.54 & $<0.01$ \\
\hline Systolic arterial pressure $(\mathrm{mmHg})$ & -0.28 & 0.71 \\
\hline Diastolic arterial pressure $(\mathrm{mmHg})$ & 0.05 & 0.42 \\
\hline Pulse pressure $(\mathrm{mmHg})$ & -0.36 & 0.02 \\
\hline Heart rate $(\mathrm{bpm})$ & -0.03 & 0.84 \\
\hline \multicolumn{3}{|l|}{ Aortic elastic properties-afterload data } \\
\hline Maximal ascending aortic diameter $(\mathrm{cm})$ & 0.10 & 0.82 \\
\hline Minimal ascending aortic diameter $(\mathrm{cm})$ & 0.26 & 0.51 \\
\hline Stiffness index, $\beta_{1}$ & -0.59 & $<0.01$ \\
\hline Systemic arterial compliance $\left(\mathrm{ml} \mathrm{mmHg}{ }^{-1} \mathrm{~m}^{-2}\right)$ & 0.54 & $<0.01$ \\
\hline Total vascular resistance $\left(\right.$ dyne $\mathrm{s} \mathrm{cm}^{-5}$ ) & -0.49 & $<0.01$ \\
\hline Aortic distensibility, $\mathrm{D},\left(\mathrm{cm}^{2}\right.$ dyne $\left.{ }^{-1} 10^{-6}\right)$ & 0.21 & 0.17 \\
\hline \multicolumn{3}{|l|}{ Aortic valve data } \\
\hline Indexed aortic valve area $\left(\mathrm{cm}^{2} / \mathrm{m}^{2}\right)$ & 0.44 & $<0.01$ \\
\hline Dimensionless velocity index & 0.34 & 0.02 \\
\hline Energy loss index $\left(\mathrm{cm}^{2} / \mathrm{m}^{2}\right)$ & 0.38 & 0.01 \\
\hline Mean aortic gradient $(\mathrm{mmHg})$ & -0.28 & 0.07 \\
\hline $\mathrm{Z}_{\mathrm{VA}}\left(\mathrm{mmHg} / \mathrm{ml} \mathrm{m}^{2}\right)$ & -0.59 & $<0.01$ \\
\hline \multicolumn{3}{|l|}{ Left ventricular data } \\
\hline Stroke volume index $\left(\mathrm{ml} / \mathrm{m}^{2}\right)$ & 0.36 & 0.02 \\
\hline LV ejection fraction by Simpson (\%) & 0.31 & 0.04 \\
\hline LV mass indexed $\left(\mathrm{g} / \mathrm{m}^{2}\right)$ & -0.12 & 0.42 \\
\hline Relative wall thickness & -0.15 & 0.32 \\
\hline Global longitudinal $\varepsilon(\%)$ & -0.16 & 0.34 \\
\hline E/e' & -0.37 & 0.01 \\
\hline
\end{tabular}

CAASR circumferential ascending aortic strain rate, $L V$ left ventricular, $Z_{V A}$ Valvulo-arterial impedance

Table 3 Model 1: Clinical parameters to predict CAASR

\begin{tabular}{lrll}
\hline Variables & $\beta$ & $T$ & $p$ \\
\hline Age (years) & -0.29 & -2.0 & 0.05 \\
Body surface area $\left(\mathrm{m}^{2}\right)$ & 0.32 & 2.13 & 0.04 \\
Pulse pressure $(\mathrm{mmHg})$ & -0.25 & -2.0 & 0.052 \\
\hline
\end{tabular}

F $9.0(p<0.01), R^{2}=0.40$

Table 4 Model 2: Afterload parameters to predict CAASR

\begin{tabular}{lrrr}
\hline Variables & \multicolumn{1}{c}{$\beta$} & \multicolumn{1}{c}{$T$} & \multicolumn{1}{l}{$p$} \\
\hline Stiffness index, $\beta_{1}$ & -0.42 & -3.4 & 0.02 \\
Systemic arterial compliance & 0.24 & 1.7 & 0.89 \\
$\quad\left(\mathrm{ml} \mathrm{mmHg}^{-1} \mathrm{~m}^{-2}\right)$ & & & \\
Total vascular resistance $($ dyne s cm & \\
\end{tabular}

F $13.0(p<0.01), R^{2}=0.49$
Table 5 Model 3: Valvular and left ventricular parameters to predict CAASR

\begin{tabular}{lrrr}
\hline Variables & $\beta$ & $T$ & $p$ \\
\hline Indexed aortic valve area $\left(\mathrm{cm}^{2} / \mathrm{m}^{2}\right)$ & 0.31 & 2.16 & 0.04 \\
Stroke volume index $\left(\mathrm{ml} / \mathrm{m}^{2}\right)$ & 0.19 & 1.34 & 0.19 \\
E/e' & -0.29 & -2.23 & 0.03 \\
\hline F 6.3 $(p<0.01), R^{2}=0.32$ & & &
\end{tabular}

F $6.3(p<0.01), R^{2}=0.32$

Table 6 Model 4: Final linear regression model to predict CAASR

\begin{tabular}{lrrr}
\hline Variables & \multicolumn{1}{c}{$\beta$} & \multicolumn{1}{c}{$T$} & \multicolumn{1}{c}{$p$} \\
\hline Age (years) & -0.25 & -1.88 & 0.07 \\
Body surface area $\left(\mathrm{m}^{2}\right)$ & 0.13 & 0.89 & 0.38 \\
Stiffness index, $\beta_{1}$ & -0.41 & -3.55 & $<0.01$ \\
Indexed aortic valve area $\left(\mathrm{cm}^{2} / \mathrm{m}^{2}\right)$ & 0.21 & 1.95 & 0.06 \\
E/e' & -0.16 & -1.26 & 0.22 \\
\hline
\end{tabular}

F $10.4(p<0.01), R^{2}=0.57$

Follow up analysis

Data was available for all 45 patients, with a median follow-up time of 955 (536-1,029) days. During this time 14 (31\%) patients died. CAASR was significantly lower for the patients who died during follow up $(0.61 \pm 0.18$ vs. $0.80 \pm 0.28 \mathrm{~s}^{-1}, p=0.03$ ); conversely, no difference was identified regarding CAAS. A similar association was noted for CAASR to estimate cardiovascular mortality. No association was found with aortic mechanics (either strain or SR) regarding other endpoints, as AVR and admission for heart failure-Table 7 .

A CAASR cutpoint of $0.66 \mathrm{~s}^{-1}$ showed $71.4 \%$ sensitivity and $64.5 \%$ specificity to predict mortality during longterm follow up (AUC, 0.70; $95 \%$ CI 0.54-0.82, $p=0.02$ ). Patients with a baseline global CAASR $>0.66 \mathrm{~s}^{-1}$ had a significant higher survival rate (83.3 vs. $52.4 \%$, Log Rank $p=0.04$ ) (Fig. 4) than patients with values $<0.66 \mathrm{~s}^{-1}$.

Utility of aortic strain rate in estimating vascular load

In 20 of our patients, $\mathrm{SAC}$ was $\leq 0.6 \mathrm{ml} / \mathrm{mmHg} / \mathrm{m}^{2}$. CAASR in these patients was significantly lower $(0.63 \pm 0.21$ vs. $\left.0.84 \pm 0.27 \mathrm{~s}^{-1}, p<0.01\right)$. In 14 of our patients, TVR was $>2,000$ dynes $/ \mathrm{sec} / \mathrm{cm}^{-5}$. CAASR in these patients was also significantly lower $\left(0.82 \pm 0.25\right.$ vs. $0.56 \pm 0.20 \mathrm{~s}^{-1}$, $p<0.01$ ). Low SAC and elevated TVR were observed together in 11 patients. These subjects had the lowest CAASR values, compared with other patient subsets where SAC and TVR values were normal, or where SAC values alone were low and TVR normal (CAASR: $0.86 \pm 0.27$, $0.74 \pm 0.19$, and $0.54 \pm 0.19 \mathrm{~s}^{-1}$, respectively; $p<0.01$ ) (Fig. 5). 

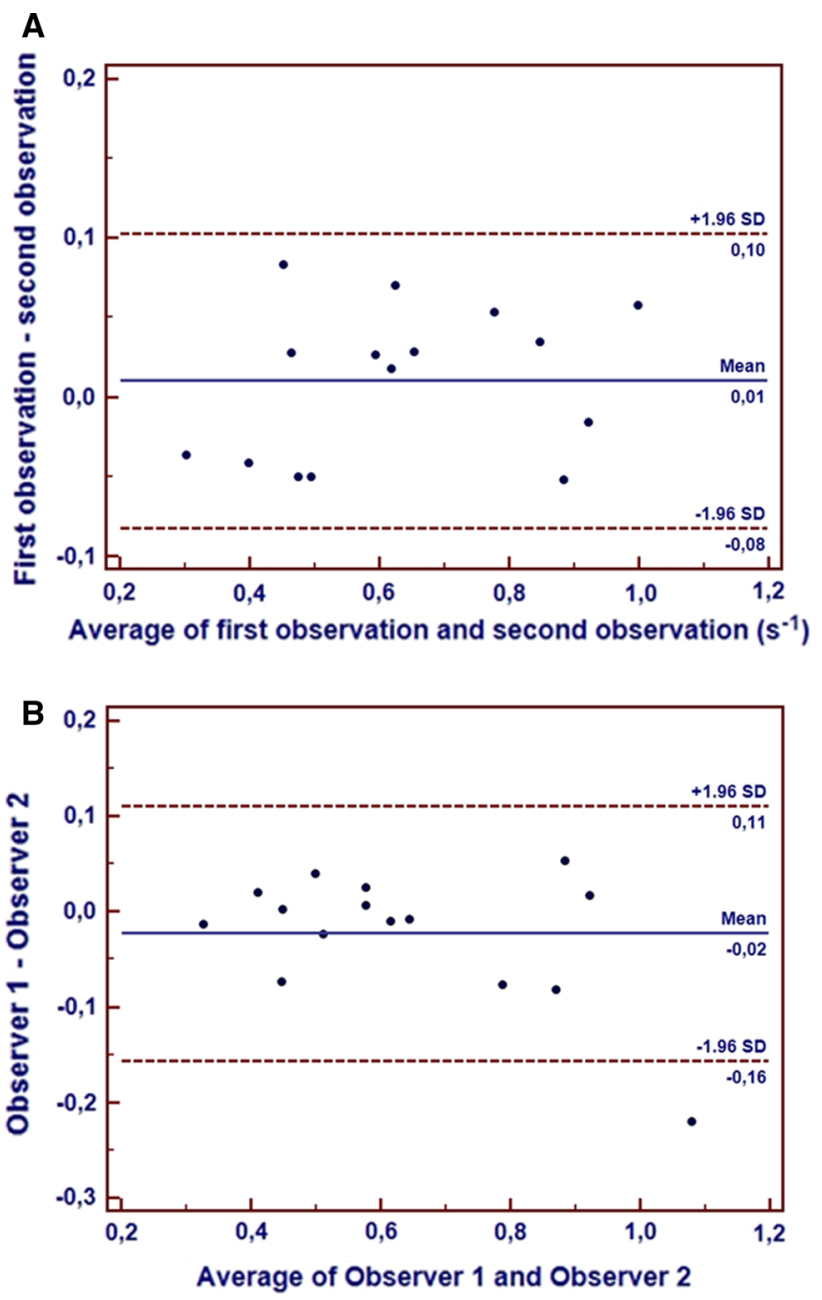

Fig. 3 a Bland-Altman plot of intra-observer global CAASR $\left(\mathrm{s}^{-1}\right)$ variability (Bias, $0.01 \mathrm{~s}^{-1}$; $95 \% \mathrm{CI}-0.08$ to $0.1 \mathrm{~s}^{-1}$ ). b BlandAltman plot of inter-observer global CAASR $\left(\mathrm{s}^{-1}\right)$ variability (Bias, $-0.02 \mathrm{~s}^{-1} ; 95 \% \mathrm{CI}-0.16$ to $0.11 \mathrm{~s}^{-1}$ )

Overall, we found that valvular and vascular components evolved in parallel. iAVA and CAASR values declined in tandem, along with increases in SVR (supplemental Table 1). However, SAC and stiffness index did not share this relationship.

\section{Discussion}

Our findings, based on 2D-ST technology, demonstrate the following concepts: (1) high feasibility and reproducibility of global CAASR determinations in patients with moderate to severe AS; (2) correlation of CAASR and multiple parameters by univariate analysis, but $\beta_{1}$ index was independently associated with CAASR; (3) association of CAASR with a SAC decline, a TVR elevation and with the LV remodeling process; (4) prognostic influence of CAASR.
Table 7 Follow up Data

\begin{tabular}{lll}
\hline Endpoints & CAASR $\left(\mathrm{s}^{-1}\right)$ & CAS $(\%)$ \\
\hline 1.Mortality & & \\
Yes $(n=14)$ & $0.61 \pm 0.18$ & $5.9 \pm 2.9$ \\
No $(n=31)$ & $0.80 \pm 0.28$ & $6.9 \pm 3.1$ \\
$p$ value & 0.028 & 0.28 \\
2.Cardiovascular mortality & & \\
Yes $(n=10)$ & $0.59 \pm 0.19$ & $5.8 \pm 3.0$ \\
No $(n=35)$ & $0.78 \pm 0.27$ & $6.4 \pm 3.0$ \\
$p$ value & 0.05 & 0.55 \\
3.Aortic valve replacement & & \\
Yes $(n=11)$ & $0.76 \pm 0.26$ & $6.0 \pm 2.7$ \\
No $(n=34)$ & $0.74 \pm 0.27$ & $6.4 \pm 3.1$ \\
$p$ value & 0.80 & 0.70 \\
4.Heart failure dmission & & \\
Yes $(n=14)$ & $0.74 \pm 0.25$ & $5.6 \pm 2.6$ \\
No $(n=31)$ & $0.75 \pm 0.27$ & $6.6 \pm 3.1$ \\
$p$ value & 0.94 & 0.34 \\
5.Combined endpoint & & $5.9 \pm 2.9$ \\
Yes $(n=29)$ & $0.73 \pm 0.25$ & $6.9 \pm 3.1$ \\
No $(n=16)$ & $0.77 \pm 0.29$ & 0.28 \\
$p$ value & 0.56 & \\
\hline
\end{tabular}

$C A A S R$ circumferential ascending aortic strain rate, $C A A S$ circumferential ascending aortic strain

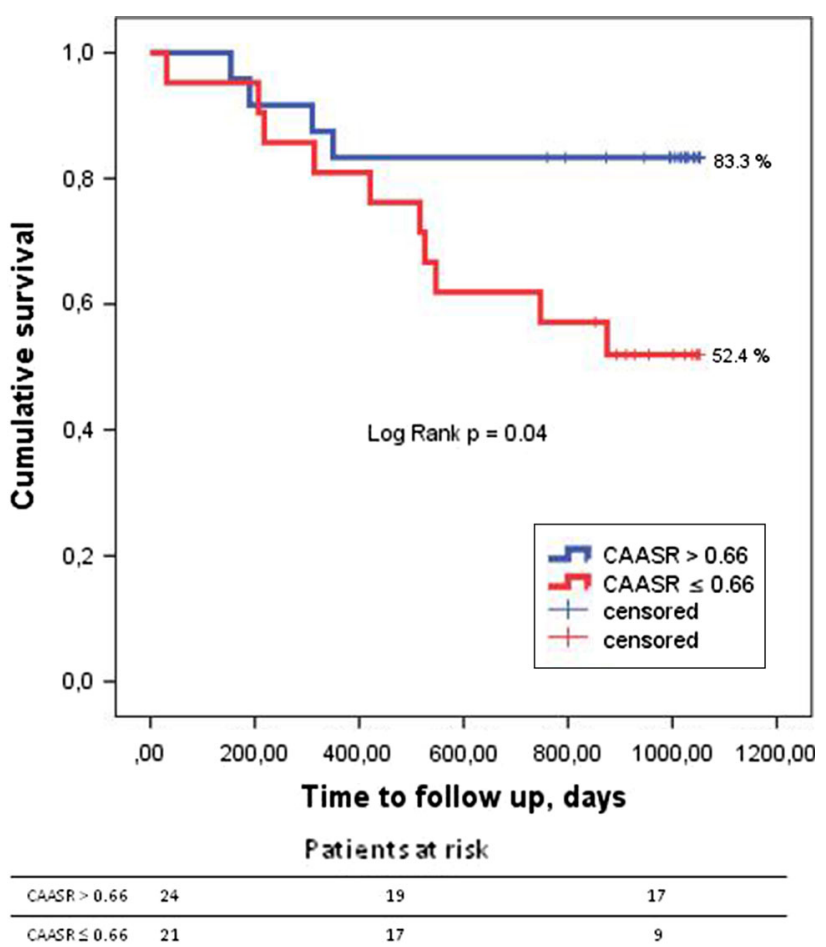

Fig. 4 Survival during long-term follow up stratified by CAASR cutpoint of $0.66 \mathrm{~s}^{-1}$ 


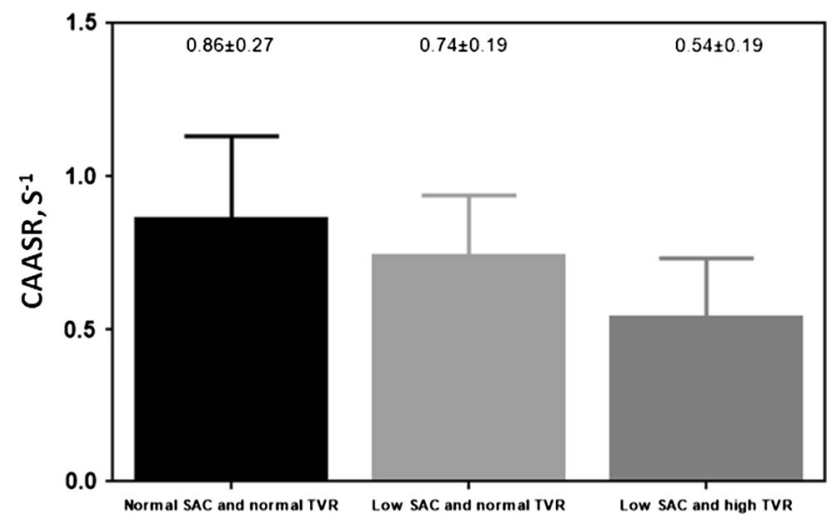

Fig. 5 CAASR in three patient subsets: normal SAC + normal TVR $(n=22)$; low SAC + normal TVR $(n=8)$; low SAC + elevated $\operatorname{TVR}(n=11)(p<0.01)$

Circumferential ascending aorta strain rate

Declining arterial elasticity is largely attributable to progressive degeneration of elastin fibers within the media of arterial walls [26]. Collagen fibers gradually increase as a consequence, promoting stiffness and thickness of vessels. Such changes are especially important in proximal aorta, which is rich in the elastin fibers needed to support each systolic impulse and to accommodate stroke volume [27]. Arterial stiffness is one of the earliest detectable manifestations of adverse structural and functional changes within vascular walls. Stiffness increases with age in relatively healthy individuals and in the presence of hypertension, diabetes, and obesity [9].

This degenerative process is then bound to influence 2D-ST echocardiographic vascular mechanics [9]. In graphic depiction of the SR curve, circumferential SR assumes an early positive value during LV systole, as vessel wall expands to accommodate vascular flow. Large arteries are thus tasked with providing adequate buffering during each ventricular contraction through arterial-ventricular coupling.

Vascular circumferential SR was first conceived by Oishi et al. [9]. The original paper explores the vascular mechanics ( $\varepsilon$ and SR values) of abdominal aorta, asserting that vascular SR not only reflects the vascular degenerative aging process but also constitutes a better index within differing age groups, compared with the $\beta_{1}$ stiffness index [9]. Other studies have supported the feasibility and utility of circumferential vascular assessment as well, especially work by Bjallmark et al. [6]. These investigators showed that in the common carotid artery, evaluation of vascular mechanics (including SR) via 2D-ST technology proved superior to conventional measures of vascular stiffness in assessing elastic properties of vessels [6]. Moreover, an important clinical implication of vascular $\varepsilon$ and SR has been demonstrated recently. Parameters of carotid arterial vascular mechanics have served to predict past history of stroke in older subjects with existing increases in vascular stiffness [28]. It has also been shown that $\varepsilon$ values of thoracic descending aorta, generated by velocity vector imaging software, are significantly lower in patients with AS, compared with values of patients with aortic regurgitation (AR); and that a bicuspid aortic valve negatively impacts aortic $\varepsilon$ value in patients with either AS or AR [29].

To the best of our knowledge, this is the first effort to assess deformation of thoracic ascending aorta in terms of vascular SR. In related research on thoracic aortic mechanics, Vitarelli et al. [11] relied on tissue Doppler imaging and radial parameters. Radial deformation assesses the process of vascular thickening, which in our opinion is not conceptually equivalent with vascular wall deformation. Others have also demonstrated the poor performance of radial deformation in predicting vascular stiffening [6]. From our data, we found that locally assessed vascular stiffness was independently associated with CAASR, supporting vascular SR as best gauge of degenerative vascular remodeling.

Is CAASR useful for patients with aortic stenosis?

It is currently acknowledged that an imbalance in LV hemodynamic load increases and the capacity to overcome such increases is responsible for adverse outcomes in AS [3]. Not only is LV afterload increased by valvular obstruction, but vascular load is similarly increased. It is also well-established that reduced systemic compliance exists in $>40 \%$ of patients with AS. This reduction in arterial compliance then exacerbates the LV afterload burden, culminating in adverse clinical events [2]. The changing face of this disease underscores a need for more comprehensive assessment of AS, beyond classic variables, such as peak jet velocity, pressure gradients, valvular area, and LV function.

Through this investigation, we have shown that CAASR may be a useful non-invasively derived variable for studying the vascular component of AS, independent of blood pressure and LV performance measures, such as stroke volume. Lower CAASR correlated with increased vascular stiffness, thus indicating a higher global LV afterload. Importantly, CAASR was associated with both a pulsatile component of arterial load (SAC) and a static one (TVR). Contrary to other studies of vascular deformation, CAASR and blood pressure were unrelated [28]. Nevertheless, we believe our data are corroborated elsewhere in medical literature, where up to one-third of patients with AS have pseudo-normalized blood pressure due to reduced SAC and superimposed LV dysfunction [2, 30]. Our data also indicate a significant correlation between CAASR, estimated LV filling pressures, and LVEF, all of which 
attest to the critical influence of vascular changes on the ventricular remodeling process, even in patients with moderate to severe AS.

In the setting of AS, we recently identified SVI as the most important determinant of circumferential ascending aortic $\varepsilon$, meaning that circumferential vascular deformation was dependent on change in vascular flow and not on local vascular wall properties [12]. Herein, we found that the vascular stiffness index $\left(\beta_{1}\right)$ was strongly associated with CAASR, suggesting that the rate of circumferential vascular deformation corresponds with local arterial rigidity. CAAS and CAASR thus are complementary parameters that may aid in the non-invasive echocardiographic assessment of stroke flow and vascular load in patients with AS.

Although the primary aim of our study was to analyse the physiological determinants of CAASR in patients with degenerative AS, as an exploratory endpoint we also we also assessed clinical outcomes. We were able to demonstrate an association of thoracic ascending aortic mechanical parameters (namely CAASR, but not CAAS) with mortality during long-term follow-up. Therefore, we suggest that future research should focus on the clinical usefulness of aortic mechanics over classic outcome prediction variables, such as AVA, LV systolic and diastolic performance, flow, and vascular load.

\section{Clinical implications}

Given the feasibility and reproducibility of 2D-ST global CAASR, we advocate its routine use in assessing the vascular loads of patients with AS. Of particular note, CAASR is a non-invasive echocardiographic parameter, unaffected by blood pressure and LV performance.

\section{Limitations}

Our analyses were based on a single centre, observational study, with a small number of patients. Brachial blood pressure was utilized, rather than central blood pressure. Brachial pressure is generally higher than central pressure, although recent data supports a reasonable clinical agreement between non-invasive brachial pressures and directly measured central aortic pressures in patients with AS [31]. To date, there is no gold standard for evaluating local arterial stiffness. As a matter of protocol, we chose vascular stiffness index [32] to validate CAASR. A recent study found no relationship between vascular mechanics and pulse wave velocity, suggesting that vascular $\varepsilon$ and SR reflected local (not global) arterial stiffness [28]. We also had no invasive data regarding cardiac output, total systemic resistance and systemic vascular compliance.

\section{Conclusions}

CAASR determination showed high feasibility and excellent reproducibility in patients with moderate to severe AS. The stiffness index was independently associated with CAASR, and it had long-term prognostic influence, making CAASR a promising tool for studying the vascular loading component of patients with AS.

Acknowledgments The authors thank Nélson Ribeiro BSc, and Ana Paula Oliveira BSc, for their help during initial echocardiographic patient evaluations, as well as Mrs Sónia Cancela for her logistic support.

Conflict of interest The authors have no conflicts of interest.

Funding None.

\section{References}

1. Rajamannan NM, Evans FJ, Aikawa E, Grande-Allen KJ, Demer LL et al (2011) Calcific aortic valve disease: not simply a degenerative process: a review and agenda for research from the National Heart and Lung and Blood Institute Aortic Stenosis Working Group. Executive summary: calcific aortic valve disease-2011 update. Circulation 124:1783-1791

2. Briand M, Dumesnil JG, Kadem L, Tongue AG, Rieu R et al (2005) Reduced systemic arterial compliance impacts significantly on left ventricular afterload and function in aortic stenosis: implications for diagnosis and treatment. J Am Coll Cardiol 46:291-298

3. Pibarot P, Dumesnil JG (2012) Improving assessment of aortic stenosis. J Am Coll Cardiol 60:169-180

4. Hamilton PK, Lockhart CJ, Quinn CE, McVeigh GE (2007) Arterial stiffness: clinical relevance, measurement and treatment. Clin Sci 113:157-170

5. Pannier BM, Avolio AP, Hoeks A, Mancia G, Takazawa K (2002) Methods and devices for measuring arterial compliance in humans. Am J Hypertens 15:743-753

6. Bjallmark A, Lind B, Peolsson M, Shahgaldi K, Brodin LA et al (2010) Ultrasonographic strain imaging is superior to conventional non-invasive measures of vascular stiffness in the detection of age-dependent differences in the mechanical properties of the common carotid artery. Eur J Echocardiogr 11:630-636

7. Becker M, Bilke E, Kuhl H, Katoh M, Kramann R et al (2006) Analysis of myocardial deformation based on pixel tracking in two dimensional echocardiographic images enables quantitative assessment of regional left ventricular function. Heart 92: 1102-1108

8. Helle-Valle T, Crosby J, Edvardsen T, Lyseggen E, Amundsen $\mathrm{BH}$ et al (2005) New noninvasive method for assessment of left ventricular rotation: speckle tracking echocardiography. Circulation 112:3149-3156

9. Oishi Y, Mizuguchi Y, Miyoshi H, Iuchi A, Nagase N et al (2008) A novel approach to assess aortic stiffness related to changes in aging using a two-dimensional strain imaging. Echocardiography 25:941-945

10. Kim KH, Park JC, Yoon HJ, Yoon NS, Hong YJ et al (2009) Usefulness of aortic strain analysis by velocity vector imaging as a new echocardiographic measure of arterial stiffness. J Am Soc Echocardiogr 22:1382-1388 
11. Vitarelli A, Giordano M, Germano G, Pergolini M, Cicconetti P et al (2010) Assessment of ascending aorta wall stiffness in hypertensive patients by tissue Doppler imaging and strain Doppler echocardiography. Heart 96:1469-1474

12. Teixeira R, Moreira N, Baptista R, Barbosa A, Martins R et al (2013) Circumferential ascending aortic strain and aortic stenosis. Eur Heart J Cardiovasc Imaging 14:631-641

13. Dubois D, Dubois E (1916) A formula to extimate the approximate surface area if height and weight are know. Arch Inter Med 17:863-871

14. Kadem L, Dumesnil JG, Rieu R, Durand LG, Garcia D et al (2005) Impact of systemic hypertension on the assessment of aortic stenosis. Heart 91:354-361

15. Evangelista A, Flachskampf F, Lancellotti P, Badano L, Aguilar R et al (2008) European Association of Echocardiography recommendations for standardization of performance, digital storage and reporting of echocardiographic studies. Eur J Echocardiogr 9:438-448

16. Lang RM, Bierig M, Devereux RB, Flachskampf FA, Foster E et al (2005) Recommendations for chamber quantification: a report from the American Society of Echocardiography's Guidelines and Standards Committee and the Chamber Quantification Writing Group, developed in conjunction with the European Association of Echocardiography, a branch of the European Society of Cardiology. J Am Soc Echocardiogr 18:1440-1463

17. Dubin J, Wallerson DC, Cody RJ, Devereux RB (1990) Comparative accuracy of Doppler echocardiographic methods for clinical stroke volume determination. Am Heart J 120:116-123

18. Nagueh SF, Appleton CP, Gillebert TC, Marino PN, Oh JK et al (2009) Recommendations for the evaluation of left ventricular diastolic function by echocardiography. J Am Soc Echocardiogr 22:107-133

19. Stefanadis C, Stratos C, Boudoulas H, Kourouklis C, Toutouzas P (1990) Distensibility of the ascending aorta: comparison of invasive and non-invasive techniques in healthy men and in men with coronary artery disease. Eur Heart J 11:990-996

20. Garcia D, Pibarot P, Dumesnil JG, Sakr F, Durand LG (2000) Assessment of aortic valve stenosis severity: a new index based on the energy loss concept. Circulation 101:765-771

21. Catalano M, Lamberti-Castronuovo A, Catalano A, Filocamo D, Zimbalatti C (2011) Two-dimensional speckle-tracking strain imaging in the assessment of mechanical properties of carotid arteries: feasibility and comparison with conventional markers of subclinical atherosclerosis. Eur J Echocardiogr 12:528-535

22. Yuda S, Kaneko R, Muranaka A, Hashimoto A, Tsuchihashi $K$ et al (2011) Quantitative measurement of circumferential carotid arterial strain by two-dimensional speckle tracking imaging in healthy subjects. Echocardiography 28:899-906

23. Mor-Avi V, Lang RM, Badano LP, Belohlavek M, Cardim NM et al (2011) Current and evolving echocardiographic techniques for the quantitative evaluation of cardiac mechanics: ASE/EAE consensus statement on methodology and indications endorsed by the Japanese Society of Echocardiography. J Am Soc Echocardiogr 24:277-313

24. Bland JM, Altman DG (1986) Statistical methods for assessing agreement between two methods of clinical measurement. Lancet $1: 307-310$

25. Shrout PE, Fleiss JL (1979) Intraclass correlations: uses in assessing rater reliability. Psychol Bull 86:420-428

26. Avolio A, Jones D, Tafazzoli-Shadpour M (1998) Quantification of alterations in structure and function of elastin in the arterial media. Hypertension 32:170-175

27. Cavalcante JL, Lima JA, Redheuil A, Al-Mallah MH (2011) Aortic stiffness: current understanding and future directions. J Am Coll Cardiol 57:1511-1522

28. Tsai WC, Sun YT, Liu YW, Ho CS, Chen JY et al (2013) Usefulness of vascular wall deformation for assessment of carotid arterial stiffness and association with previous stroke in elderly. Am J Hypertens 26:770-777

29. Petrini J, Jenner J, Rickenlund A, Eriksson P, Franco-Cereceda A et al (2014) Elastic properties of the descending aorta in patients with a bicuspid or tricuspid aortic valve and aortic valvular disease. J Am Soc Echocardiogr 27:393-404

30. Hachicha Z, Dumesnil JG, Bogaty P, Pibarot P (2007) Paradoxical low-flow, low-gradient severe aortic stenosis despite preserved ejection fraction is associated with higher afterload and reduced survival. Circulation 115:2856-2864

31. Rajani R, Chowienczyk P, Redwood S, Guilcher A, Chambers JB (2008) The noninvasive estimation of central aortic blood pressure in patients with aortic stenosis. J Hypertens 26:2381-2388

32. O'Rourke MF, Staessen JA, Vlachopoulos C, Duprez D, Plante GE (2002) Clinical applications of arterial stiffness; definitions and reference values. Am J Hypertens 15:426-444 